\title{
Prospects for Engineering Science and Technology Development Strategy Research in China
}

\author{
Yang Wei \\ National Natural Science Foundation of China, Beijing 100085, China
}

\begin{abstract}
China's engineering science and technology development strategy research is a joint project organized by the Chinese Academy of Engineering and the National Natural Science Foundation of China. The purposes of this research are to build a high-level national engineering science and technology think tank, to build a Chinese science and technology planning platform, and to develop science and technology planning in a manner that suits the Chinese national condition. This paper describes the level of Chinese science and technology expected in 2035, using top-level design and two operating systems (an administrative line system to ensure the efficiency of the strategy research and a technical line system to ensure the quality of the strategy research), to achieve the desired results.
\end{abstract}

Keywords: engineering science and technology development; strategy research; science and technology planning; technology foresight

Today's meeting marks the official launch of the Research on China's Engineering Science and Technology Development Strategy 2035, a project supported by the Chinese Academy of Engineering (CAE) and the National Natural Science Foundation of China (NSFC). All the academicians, experts, and relevant managers present today have provided constructive suggestions and recommendations for the project, which are of significant benefit to its future implementation and further improvement. I would like to offer several suggestions regarding this project.

My first suggestion is that we develop a deeper understanding of the significance of the Research on China's Engineering Science and Technology Development Strategy 2035. Since the reform and opening-up of China, we have accomplished the construction of a large number of infrastructural and worldclass projects, including the Three Gorges Hydroelectric Project (TGHP), the South-North Water Diversion Project, "super long and super high" bridges, the high-speed railway system, and "super long" tunnels. These achievements have made our country a global construction center. The next 20 years, from 2015 to 2035 , are expected to be crucial to the development of engineering technology in China. Significant and disruptive changes shall be witnessed throughout this 20-year period. By 2020, we aim to become one of the world's most innovative countries. By 2025, we expect China's gross domestic product to exceed that of the United States to become the world's largest economy. By 2030, carbon emissions in China shall reach its peak, which will have a significant impact on our energy structure. By 2035, the development of engineering science and technology in China will shift from that of a follower to being on par with that of the United States and, eventually, leading development to become the source of innovation in the fields of engineering science and engineering technology. By that point, the structure of courses on science and engineering at major universities in China will be very different than now, whereby substantial research and development tasks in the field of engineering science work will be transferred to enterprises, which will become the main body of innovation in this field. Why is such a shift occurring in China? Over the next 20 years in China, industrialization is expected to undergo a shift from incremental to disruptive change. The huge economic and social demands incurred during this process shall create a unique condition and a golden opportunity for the development of engineering science and technology.

Corresponding author: Yang Wei, Director of the National Natural Science Foundation of China and an Academician of Chinese Academy of Sciences. This article is Mr. Yang's speech at the initial meeting of the Research on China's Engineering Science and Technology Development Strategy 2035 (March 26, 2015). Chinese version: Strategic Study of CAE 2017, 19 (1): 001-003

Cited item: Yang Wei. Prospects for Engineering Science and Technology Development Strategy Research in China. Strategic Study of CAE, https://doi.org /10.15302/ J-SSCAE-2017.01.001 
First, China will be the final battlefield for traditional engineering. More specifically, the upgrade of traditional industries and construction of infrastructure still demands the development of mechanical engineering, civil engineering, chemical engineering, and electrical machinery in China. Although the United States remains a powerful player in the field of transportation equipment, China has developed sufficient technologies to challenge the United States' dominant position. Moreover, China has been leading globally in the field of metallurgy. Second, informatization will be the primary driver of China's industrialization. Industry 4.0, characterized by smart production, will accelerate the fourth industrial revolution, with a closer combination of manufacturing and information industries and a deeper integration of industrialization and informatization. Artificial intelligence and related engineering fields shall usher in a new round of development opportunities. Over the next 20 years, the demographic dividend in China will gradually disappear, the aging society will spawn the popularity of service-oriented robots, big data will play a significant role in the process of urbanization, and the networks of space, land, and ocean will become fully consolidated. All these changes will generate room for the development of information science, technology, and engineering. Third, China will become the birthplace of strategic emerging industries. China has a large population, and since the reform, private wealth has accumulated rapidly. During the transformation from a society having only adequate food and clothing to a welloff society, the increasing demand for high-quality and personalized goods generated by the growing population of the middle class is expected to create a promising consumer market that will attract the attention and envy of the world. In addition, this market shall directly develop the global consumer market and related industries, as well as lay a solid foundation for the formation and development of strategic emerging industries. Fourth, China is expected to become the new frontier and main battlefield in the fields of ecology, energy, natural resources, and the environment. Over the next 20 years, China will generate huge demand in shale gas development, carbon emissions reduction, nuclear energy utilization, water pollution control, and soil remediation, which will provide prime development opportunities for energy conservation and environmental protection, healthcare, transportation industries, and other related engineering areas. Fifth, the modernization of national defense will drive the development of aerospace technology and engineering, putting forward greater and higher requirements on the field of engineering science and technology. The next 20 years until 2035 are a critical period for the development of engineering science and technology in China; therefore, a comprehensive and systematic study of its development strategy is particularly important.

My second suggestion is that Research on China's Engineering Science and Technology Development Strategy 2035 should become an important foundation for medium- to long-term scientific and technological planning of the country. Every five years, the CAE organizes a study of China's engineering science and technology development strategy for the subsequent 20 years. Since 2009, this task has been jointly organized by the CAE and NSFC. Since the 18th National Congress of the Communist Party, China accelerated the implementation of innovationdriven development strategies. Both science and technology sectors, and the relevant government departments, have full confidence in the "new normal," whereby economic development will be led by the development of science and technology. A new medium- to long-term plan for scientific and technological development will be developed during the 13th Five-Year Plan period The Research on China's Engineering Science and Technology Development Strategy toward 2035 could become an important basis for national science and technology planning.

Science and technology planning is a science itself. Questions such as "when to plan," "what to plan," and "how to plan" require careful study and analysis. Generally, planning studies include four dimensions: improvement (specifying the aspects to be improved based on the existing situation), progression (outlining the road map for future development), expansion (designing the plan based on demand, probability, and feasibility), and originality (illustrating the disruptive contributions and potential risks). Based on my personal observation of the development of engineering science and technology over the past 10 years, as well as the prospect for the coming 20 years, planning should not ignore the crucial transformations in the development of engineering science and technology in China, including from re-innovation to leap-forward innovation (such as the Tianhe supercomputer), from process innovation to original innovation (such as quantum communication), from improvement innovation to innovation of system design (such as coal-to-olefin), from application innovation to generic innovation of the cornerstone (such as Internet financing), from participating in trends to formulating the rules of the field (such as mobile communication), and from tracking innovation to efficiency innovation (such as shield tunneling machines).

How should we plan the strategy for the next 20 years of engineering science and technology development when faced with highly innovative development with Chinese characteristics? If we liken the development of engineering science and technology to hiking, five possible situations may occur during the process. In the first situation, the trail ahead is obviously visible, which means that we have a clear understanding of the requirements and pathways. In this situation, we can draw a path and design a preliminary development route based on the maturity level of the current technology. In the second situation, the trail is indistinct, such that we cannot see the entire route; hence, there could be paths ahead of us that we cannot walk down. In this situation, we should uncover the obstacles or blocks through careful planning and find a way to solve the problems. In the third situation, the trail winds along mountain ridges and the originally planned route is no longer applicable. In this situation, 
we must design new plans that include solutions to various possible situations. In the fourth situation, the road leads to a deadend. In this situation, we have to return to the starting point, redesign the pathways, and find a new route. The fifth situation involves a catastrophic route. Even if the trail does not lead to a dead-end, taking the path may lead to a disastrous impact on the economy and society, such as over-development that damages diversity or setting an unreasonable goal that we are unlikely to achieve, thereby damaging the credibility of the government and the science and technology community. A typical example is the catastrophic impact that the current extensive development has had on natural resources and the environment. Therefore, studies on engineering and technology planning should provide additional focus on the potential negative influences of the development and avoid the possibility of causing damage through the overall design. In addition, experts in humanities and social sciences should be invited to participate in the planning process. Essentially, the emergence of situations during the planning process itself is also possible. However, I believe that through an in-depth study of China's Engineering Science and Technology Development Strategy 2035, we should be able to outline the blueprint for the development of engineering science and technology for the coming 20 years and provide a valuable reference to the overall scientific and technological development planning in China.

My third suggestion is that scientific and systematic approaches should be adopted for Research on China's Engineering Science and Technology Development Strategy 2035. Science and technology planning is a science. Particularly, over the past 30 years, sophisticated methods have been developed globally for the identification and selection of priorities in research and development, as well as the prediction of the future orientation of science and technology development. In addition, scholars and practitioners have been continuously exploring new and more effective systematic methods. According to the previous introductions of corresponding work groups and representatives of the China Academy of Strategy on Aerospace Engineering Science and Technology (CAEST), Research on China's Engineering Science and Technology Development Strategy 2035 has included several common approaches in scientific and technological planning, including technology foresight, scientific metrology, and technology roadmaps. It is well-known that based on the principles developed through science and technology, economics, and social development as well as their interactions, technology foresight employs a series of normative methods, such as scenario analysis, brainstorming, and the Delphi method to systematically project the trends of coordinated development between science and technology, and the economy and society, over the next 10 to 30 years. The purpose is to identify strategic research areas and emerging general-purpose technologies that may produce the greatest economic and social benefits, thereby creating a list of alternative key technologies and major scientific fields.
Technology foresight was originally developed by the United States during the Cold War era. However, it was not until around 1970, when competition between the world's leading economies became increasingly intense, that countries such as Japan, Germany, and the United Kingdom began to apply technical foresight techniques to the early identification of potential demand for future economic and social development. The technology foresight process in Japan, which is implemented once every five years, constitutes one of the most well-known projects of its kind. Up to now, Japan has successfully completed nine rounds of technology foresight and is currently conducting its 10th round; the methods used in their technology foresight process have continuously improved and the results have been updated. The results of the Delphi surveys initially launched by other countries were generally compared to the results of the technology foresight process of Japan. We are pleased to see that, in addition to introducing the experience and achievements of Japan and other developed countries, the implementation plan for the Research on China's Engineering Science and Technology Development Strategy 2035 proposed by the CAEST also incorporates sophisticated methodology and preliminary achievements from previous technology foresight events conducted by the CAS and the Ministry of Science and Technology.

CAEST has rich experience in organizing research on scientific and technological planning and strategies. Aerospace tasks are characterized as those that have a distinctive overall design of organization and implementation at the top level that guides specific research implementation allocated to corresponding sub-systems. The proposed implementation plan is a fine representation of such characteristics. It can be observed from the implementation plan that the organization of strategy research is divided into two major systems: the administrative line system and technical line system. The administrative line system ensures the efficiency of strategy research, and the technical line system guarantees its quality. I opine that the implementation plan has fully considered the integration of the two systems and should be able to ensure the desired results of strategy research. Simultaneously, I sincerely hope that this research project becomes a longterm practice, similar to Japan, which will not only serve future science and technology planning in China but will also help us to become the world's leading technology foresight project.

The NSFC and the CAE have had several years of experience cooperating in strategy research over the medium- to long-term development of China's engineering science and technology fields. In the past years, the two organizations have mainly supported bottom-up individual projects; however, strategy research moving forward to the year 2035 will shift towards top-down systematic research projects. We are very pleased to continue our support and participation in this important task. Through strategy research, I hope that the NSFC and the CAE will jointly establish a high-level national engineering science and technology think tank, build an engineering planning platform that integrates various resources, 
such as data, analysis, modeling, knowledge library, roadmaps, and data sources, as well as develop planning studies for science and technology that is suited to the situation in China.

The NSFC is currently formulating its "13th Five-Year" development-planning program. Although basic research involves more uncertainty in comparison to engineering science and technology, the planning of basic research may be more challenging; we hope that academicians in the field of engineering science and technology become an important part of the NSFC think tank. We also hope that NSFC's strategy research and the strategy research of engineering science and technology can be soundly integrated and that the funds from the NSFC provide a strong support to the development of engineering science and technology in China. 